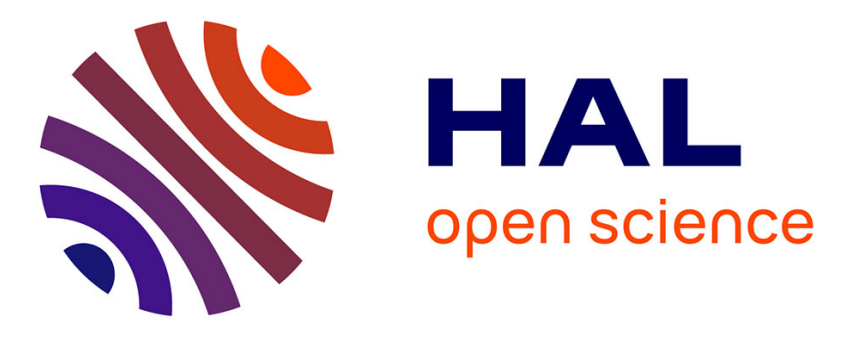

\title{
Rationalizing the formation of binary mixed thiol self-assembled monolayers
}

Douga Nassoko, Mahamadou Seydou, Claire Goldmann, Corinne Chanéac, Clément Sanchez, David Portehault, Frederik Tielens

\section{- To cite this version:}

Douga Nassoko, Mahamadou Seydou, Claire Goldmann, Corinne Chanéac, Clément Sanchez, et al.. Rationalizing the formation of binary mixed thiol self-assembled monolayers. Materials Today Chemistry, 2017, 5, pp.34-42. 10.1016/j.mtchem.2017.05.002 . hal-01540333

\section{HAL Id: hal-01540333 https: / hal.sorbonne-universite.fr/hal-01540333}

Submitted on 16 Jun 2017

HAL is a multi-disciplinary open access archive for the deposit and dissemination of scientific research documents, whether they are published or not. The documents may come from teaching and research institutions in France or abroad, or from public or private research centers.
L'archive ouverte pluridisciplinaire HAL, est destinée au dépôt et à la diffusion de documents scientifiques de niveau recherche, publiés ou non, émanant des établissements d'enseignement et de recherche français ou étrangers, des laboratoires publics ou privés. 


\title{
Rationalizing the Formation of Binary Mixed Thiol Self- Assembled Monolayers
}

\author{
Douga Nassoko ${ }^{1,3}$, Mahamadou Seydou ${ }^{2}$, Claire Goldmann ${ }^{1}$, Corinne Chanéac ${ }^{1}$, \\ Clément Sanchez ${ }^{1}$, David Portehault ${ }^{1}$, Frederik Tielens ${ }^{1,}{ }^{*}$
}

${ }^{1}$ Sorbonne Universités, UPMC Univ Paris 06, CNRS, Collège de France, Laboratoire de Chimie de la Matière Condensée de Paris, 4 Place Jussieu, 75005 Paris, France

2 Université Paris Diderot, Sorbonne Paris Cité, ITODYS, UMR 7086 CNRS, 15 rue J.-A. de Baïf, 75205 Paris cedex 13, France.

${ }^{3}$ Ecole Normale Supérieure, Rue du 22 Octobre 1946, Quartier du Fleuve, BP:241 Bamako, Mali

Correspondence to: Dr. Frederik Tielens (E-mail: Frederik.tielens@upmc.fr) 


\begin{abstract}
Periodic DFT-D calculations are used to decipher the role of intermolecular forces on the stability of mixed linear thiol self-assembled monolayers (SAMs) on $\mathrm{Au}(111)$ and compared with experiment. The interaction energy is rationalized by quantifying its different contributions. The inter-chain interaction energy is shown to be in direct relation with the surface reconstruction and the formation of adatoms. The stability of the mixed SAM systems is predicted by calculations and validated with experiments. In order to describe predictively the segregation of binary thiol mixtures adsorption on Au surfaces a segregation descriptor is defined. This procedure is a promising step forward in the prediction of segregated SAMs leading to future functional nanomaterials, including Janus or patchy nanoparticles for optics, formulation and selfassembled patterns.
\end{abstract}

Keywords: gold nanoparticles; nano domains; Self Assembly; DFT; Thiols 


\section{Introduction}

Special efforts has been made to form in a controlled manner two-component monolayers, as a way toward adjusted surface functionalities for biocompatibility, biosensor, or molecular electronics. STM, the most used technique to characterize flat mixed SAMs, inferred that phase separation exists[1-3]. The subdomain compositions are very dependent on the chain type of each component and on the adsorption conditions (variation of temperature and concentration); Bain et al. described the co-adsorption of short- and long-chain alkanethiols on gold.[4] They described the formation of microscopic subdomains and, for the first time, pointed out the fact that the composition of the SAM may not be that in solution, $[5,6]$ which was also observed in the first characterization by DFT calculations of a mixed thiol system on gold surface.[7] Note that mixed SAMs, often formed at room temperature, are most of the time, when conformational entropy does not dominate[8], not completely at equilibrium; phase separated domains appear where each component has the average structure of pure SAMs.[9] STM studies showed that phase segregation is strongly facilitated by different thiol chain lengths. Nevertheless, at $60^{\circ} \mathrm{C}$, exchanges with the solution were shown to favour single phase islands of one thiolate.[6]

It has been shown recently that the phase separation in certain mixed SAMs can also be studied by high resolution X-ray photoemission.[10]

Drastically different chemical or physical properties as well as directionality within a single particle[11], lead to particles with fascinating hierarchical super structures[12] in solution, which tackle demanding problems in materials science, biomedicine and in the field of highly specific sensors.[13-21]

The bicomponent organic monolayers can self-assemble into nano-domains on the surface of planar substrates, where the two types of complexing agents are chemically incompatible. Similar behaviour is observed in nanoparticles, where segregation of ligands leads to new arrangements[22].

Recently, a great number of groups, worldwide, have been investigating not only the changes in the chemical and physical properties through experimental techniques but also 
using theoretical and computational approaches. Computationally much attention has been paid simulating these systems based on both Monte Carlo (MC) and molecular dynamics (MD) techniques[23-26]. For instance, Tarazona and coworkers[27] have proposed one of the first microscopic models to study the amphiphilic Janus particles from a theoretical point of view. But also other theoretical works were undertaken in this field, e.g. on interfacial nanoparticle patterning[28], and on the Self-Assembly of a Janus Polyoxometalate-Silsesquioxane (POM-POSS) Co-Cluster[29].

The organization of thiol chains on surfaces can be rationalized by means of quantum chemical calculations. The calculation of the adsorption energy per chain can provide information on the stability of the assembly formed, but also describe and quantify the physical nature of the interactions at play between the chains and between the chains and the surface. The present state-of-the-art calculated adsorption energies can be decomposed and analyzed as it has been done before in the case of amino acid monolayer assemblies.[30-34]

It should be noted that the adsorption of thiol molecules cannot be generalized to one model, since the polarization of the sulphur atom is very sensible to the thiol chain; at least this is what we would like to show in results presented here. This means that the adsorption process involving S-H bond breaking[35-38] and or adatom adsorption[39-42] is expected to be dependent of the type of thiol.

In the present paper we present for the first time a study on the rationalization of the mixed monolayer assembly pattern formation on the basis of ab initio/DFT-D interaction energies. In particular the adsorption energy decomposition is used to investigate the stability of assemblies formed by a large series of very different thiols, never been investigated at such a level of calculation. This strategy enables studying the segregation, which is quantified and predicted by introducing a descriptor based on the thiol/thiol DFT-based interaction energies.

\section{Computational Details}

\section{Calculation level}


All calculations were performed using the ab initio plane-wave pseudopotential approach as implemented in the VASP code[43, 44]. The Perdew-Burke-Ernzerhof (PBE) functional $[45,46]$ was chosen to perform the periodic DFT calculations with an accuracy on the overall convergence tested elsewhere[47-52]. The valence electrons were treated explicitly and their interactions with the ionic cores are described by the Projector Augmented-Wave method (PAW), $[44,53,54]$ which allows to use a low energy cut off equal to $400 \mathrm{eV}$ for the plane-wave basis. The integration over the Brillouin zone was performed on $2 \times 5 \times 1 \mathrm{k}$-point mesh. Open shell calculations were performed of the thiol radicals.

In the geometry optimizations at $0 \mathrm{~K}$, the positions of all atoms in the supercell are relaxed in the potential energy determined by the full quantum mechanical electronic structure until the total energy differences between the loops is less than $10^{-4} \mathrm{eV}$.

In order to account for the dispersion interaction in the SAM system, DFT-D2 approach of Grimme[55] and DFT-D3[56] were used, as implemented in VASP, which consists in adding a semi-empirical dispersion potential to the conventional Kohn-Sham DFT energy. In the original paper of Grimme (D2 correction) only the first two rows of the periodic table of elements are included - for gold we used a value of $40.62 \mathrm{~J} . \mathrm{nm}^{6} / \mathrm{mol}$ for the dispersion coefficient C6 and of $1.772 \AA$ for the van der Waals radius (RO)[57, 58]. For the D3 correction the default parameters where used.

\section{Description of the model}

The model used to study the surfaces of the gold nano-particles is approximated to the stable densely packed $\mathrm{Au}(111)$ surface, which as is shown here is not only a choice based on the constraints due to computational power available today but also because the $\mathrm{Au}(111)$ surface is a widely used model surface studied in surface science and quantum chemical calculations. The alkanethiolate SAM structure on $\mathrm{Au}(111)$ consists of an ordered $c(4 \times 2)$ super lattice containing 4 chains in both gas phase and liquid environments $[59,60]$. In these lattices, the alkanethiolate molecules are chemisorbed on the Au surface by their S-tails forming a thiolate bond, having tilt angles ranging from $200-40 \circ$ with respect to 
the substrate normal. The chemisorbed alkylthiolate chains adsorb as a $c(4 \times 2)$ pattern containing four thiol chains, being a little more stable than the $(\sqrt{ } 3 \times \sqrt{ } 3) \mathrm{R} 30$ o unit cell containing one chain. It is this unit cell which was used in the present study. This supercell may contain combinations of differently oriented chains, making patterns all within a few $\mathrm{kJ} / \mathrm{mol}$.[38, 61] However, most of them were derived for short chain thiols and were not compared to experimental structural data to convincingly select the preferred model. This study does not pretend to investigate all possible closed packed superstructures, but the existing interactions between the different thiol chains. Since the thiol models have different relative geometries possible within a window of few $\mathrm{kJ} / \mathrm{mol}$ we have chosen to simulate the surface with a $(\sqrt{ } 3 \times 2 \sqrt{ } 3) \mathrm{R} 30$ o unit cell containing two thiol molecules. This choice emerges from a price/quality point of view. The surface layer was modelled by a slab consisting of 5 hexagonal layers with 6 atoms each, as was used with success before[35, 62]. In the past several studies have been undertaken in order to investigate the adsorption site of thiol chains on $\mathrm{Au}(111)$. The adsorption site is taken to be on the displaced bridge[62], without considering any possible drastic reconstruction of the gold surface, such as the formation of gold atoms. The adsorbed thiolates are modelled by their respective thiyl radicals with an open shell electronic structure. Within this $(\sqrt{ } 3 \times 2 \sqrt{ } 3) \mathrm{R} 30$ ㅇ unit cell two thiolates are chemisorbed on the Au slab. In order to focus on the effect of the intermolecular interactions, expected to be the key in the thermodynamic stability of mixed thiol SAMs, all thiols studied were adsorbed on the same unreconstructed $\mathrm{Au}(111)$ surface. This will enable us to investigate on the same basis the surface reconstruction, leading to the formation of adatoms or not. It should be stressed that it is not because one example of thiols adsorbed better on an adatom and/or form adatoms that all thiols behave similarly. A typical extrapolation seen in literature is the case of methyl thiol that is often used as model in thiol SAM studies, but which is not representative for all types of thiols. This point is one of the questions we would discuss in this paper. Different groups of thiols should be studied to find out the specific adsorption process associated to these different types of thiols. 
A series of seven different thiolates (See Table 1) was built using the Modelview software[63] (See Fig. 1 for the model structures). The thiolates were chosen on the basis of their practical use in experiments and applications, as well as their large diversity in physicochemical properties.

From these thiolates a selection of 20 different thiol pairs, were investigated, from which 13 containing two different thiolates per pairs in the $(\sqrt{ } 3 \times 2 \sqrt{ } 3) R 30$ unit cell. The 13 mixed pairs are (See Fig. 2): $\mathrm{C} 12 / \mathrm{C} 3 \mathrm{COOH}, \mathrm{C} 12 / \mathrm{C} 11 \mathrm{COOH}, \mathrm{C} 15 \mathrm{COOH} / \mathrm{C} 3 \mathrm{COOH}, \mathrm{Eg} / \mathrm{C} 12$, TriEg/C3COOH, TriEg/C11COOH, TriEg/C15COOH, TriEg/C12, Eg/C15COOH, Eg/C11COOH, $\mathrm{C} 5 \mathrm{CONHCH} 3 / \mathrm{C} 12, \mathrm{C} 5 \mathrm{CONHCH} 3 / \mathrm{C} 3 \mathrm{COOH}$

The segregation of the thiol chains is compared with in-house synthesized nano-particles containing facets that in first approximation can be described as $\mathrm{Au}(111)$ surface.

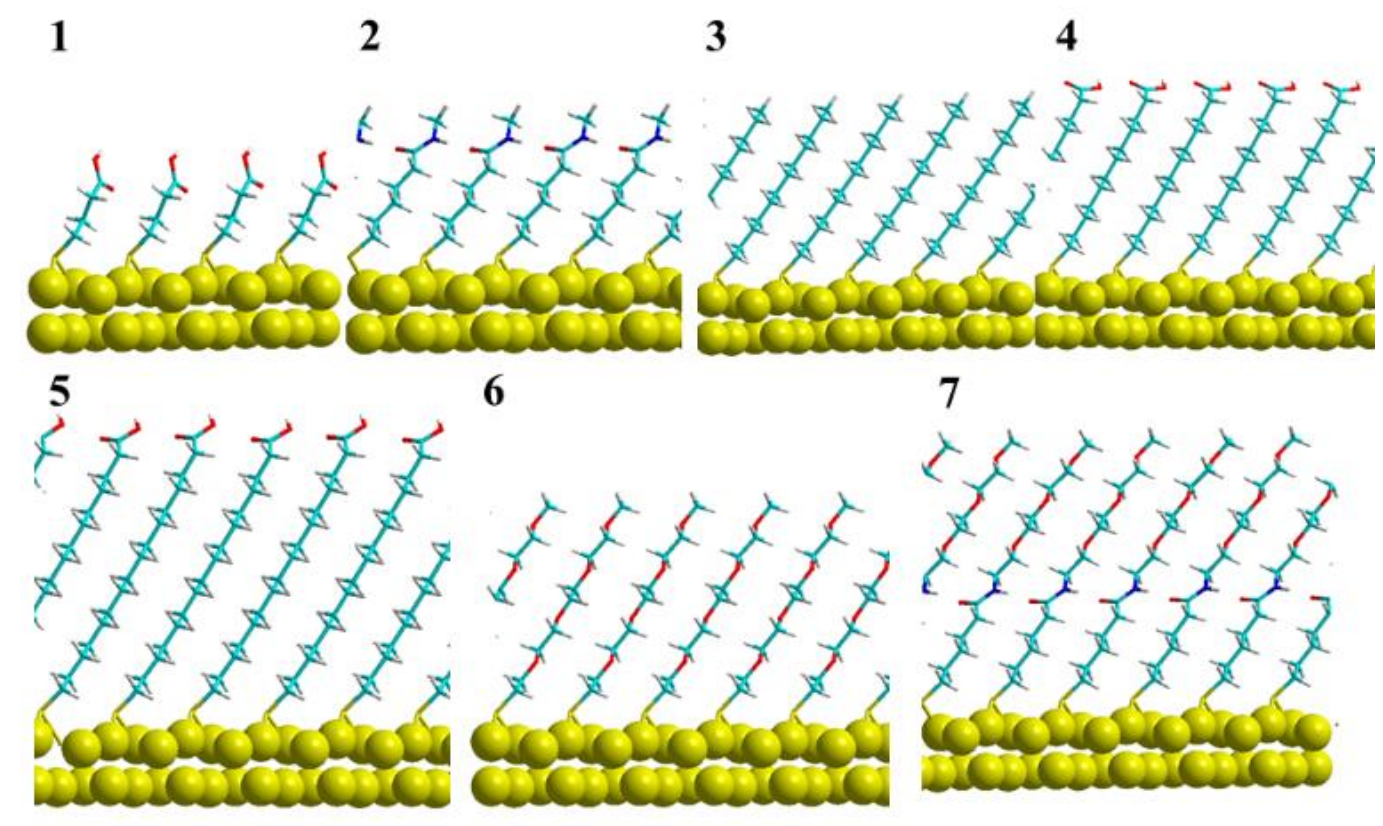

Figure 1. Side view of the optimized geometries of the pure thiol SAM systems: 1: 2C3COOH, 2: $2 \mathrm{C} 5 \mathrm{CONHCH} 3,3: 2 \mathrm{C} 12,4: 2 \mathrm{C} 15 \mathrm{COOH}, 5: 2 \mathrm{C} 11 \mathrm{COOH}, 6: 2 \mathrm{Eg}$, 7:TriEg. (Large yellow beads: gold, yellow: sulphur, light blue: carbon: white: hydrogen, blue: nitrogen, red: oxygen) 

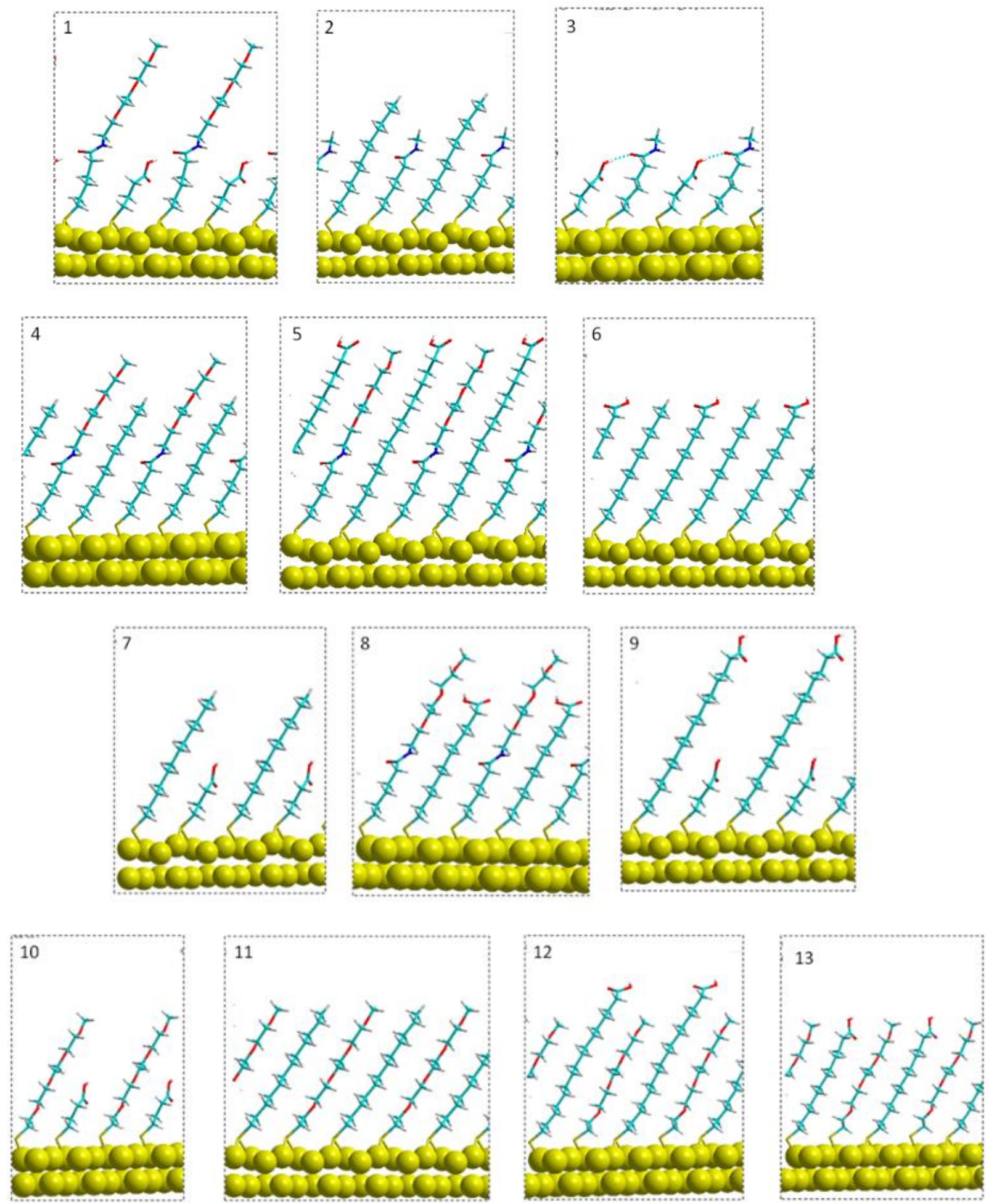

Figure 2. Side view of the optimized geometry of the mixed thiol SAM systems: 1: TriEg/C3COOH, 2:C5CONHCH3/C12, 3:C5CONHCH3/C3COOH, 4: TriEg/C12, 5 
TriEg/C3COOH, 6:C12/C11COOH, 7: $\mathrm{C} 12 / \mathrm{C} 3 \mathrm{COOH}, \quad 8: \quad T r i E g / C 11 \mathrm{COOH}, \quad 9:$ $\mathrm{C} 15 \mathrm{COOH} / \mathrm{C} 3 \mathrm{COOH}, 10: \mathrm{Eg} / \mathrm{C} 3 \mathrm{COOH}, 11: \mathrm{C} 12 / \mathrm{Eg}, 12: \mathrm{C} 15 \mathrm{COOH} / \mathrm{Eg}, 13: \mathrm{C} 11 \mathrm{COOH} / \mathrm{Eg}$. (Large yellow beads: gold, yellow: sulphur, light blue: carbon: white: hydrogen, blue: nitrogen, red: oxygen)

\section{Rationalization of the adsorption energy}

The adsorption energy $\Delta$ Eads per chain of the thiol derivate on the surface is calculated as follows:

$\Delta \mathrm{E}_{\mathrm{ads}}=\Delta \mathrm{E}_{\mathrm{ads} . \text { PBE }}+\Delta \mathrm{E}_{\mathrm{disp}}$

where $\Delta_{\text {Eads,PBE }}$ is the adsorption energy calculated from the pure DFT-PBE electronic energies. $\Delta E_{a d s, P B E}$ is calculated according to the following equation:

$\Delta E_{\text {ads }, P B E}=1 / 2[E($ thiol $A B / A u(111))-E($ thiolA $)-E($ thiolB $)-E(A u(111))]$

$E($ thiol $A B / A u(111)), E($ thiol $A), E($ thiol $B)$, and $E(A u(111))$ are the total electronic energies (at the PBE level) of the adsorption complex formed by thiolA and thiolB, the isolated thiolA and thiolB under their radical form ( 2 times the same thiol in the case of a pure SAM, since there a two chains in the unit cell), and the $A u(111)$ slab, obtained after separate geometry optimization, respectively.

$\Delta \mathrm{E}_{\text {disp }}$ is the dispersion interaction energy per chain (i.e. $1 / 2$ de dispersion interaction energy of the unit cell containing two thiol chains) calculated from a unit cell containing two thiol chains by means of the DFT-D approach[64], i.e.:

$\Delta \mathrm{E}_{\text {disp }}=\left(\Delta \mathrm{E}_{\text {ads.UC }}-\Delta \mathrm{E}_{\text {ads.PBE.UC }}\right) / 2$

with $\Delta \mathrm{E}_{\text {ads.UC }}$ and $\Delta \mathrm{E}_{\text {ads.PBE.UC }}$, the DFT-D adsorption energy and pure PBE adsorption energy of two thiol chains in the unit cell, respectively 
$\Delta$ Eads is then the adsorption energy of one thiolA chain in a perfectly ordered SAM calculated in a unit cell containing two thiol chains.

The Au-S binding energy $\Delta$ Ebind is evaluated as follows:

$\Delta E_{\text {bind }}=1 / 2 .[E($ thiolA $/ A u(111))-E($ thiolA.SAM $)-E(A u(111))]$

with E(thiolA.SAM) the single point (not geometrically relaxed) electronic energy of the thiol radicals ( 2 in the $(2 \sqrt{ } 3 \times \sqrt{ } 3) R 30$ o unit cell) in the configuration of the SAM but without considering the $\mathrm{Au}(111)$ slab.

With this $\Delta \mathrm{E}_{\text {bind }}$ one can obtain an approximation for the inter-chain interaction energy $\Delta_{\text {Eint.chain }}$ as follows:

$\Delta \mathrm{E}_{\text {int.chain }}=\Delta \mathrm{E}_{\mathrm{ads}}-\Delta \mathrm{E}_{\text {bind }}$

Another descriptor is the reconstruction energy or deformation energy per thiol in a double unit cell, defined as:

$\Delta E_{\text {recon }}=1 / 2\left(E_{\text {slab-in-SAM }}-E_{\text {clean-slab }}\right)$

Where $E_{\text {slab-in-SAM }}$ is the energy of the gold slab underneath the SAM (after relaxation) and $E_{\text {clean-slab }}$ is the energy of the pristine gold.

In order to study the stability of the thiol binary mixture a segregation descriptor $(\Xi)$ is defined:

$\Xi=\Delta \Delta \mathrm{E}=\left(\Delta \mathrm{E}_{\mathrm{ads}}-\Delta_{\mathrm{Eads}, \text { mean }}\right)$ 
The adsorption energy $\Delta \mathrm{E}_{\mathrm{ads}}$ of the binary thiolA-thiolB SAM is subtracted by the mean adsorption energy $\Delta \mathrm{E}_{\mathrm{ads}}$, mean of the pure SAMs:

$\Delta \mathrm{E}_{\mathrm{ads}, \text { mean }}=\left(\Delta \mathrm{E}_{\mathrm{ads}}, 2 \mathrm{~A}+\Delta \mathrm{E}_{\mathrm{ads}}, 2 \mathrm{~B}\right) / 2(8)$

With $\Delta \mathrm{E}_{\mathrm{ads}}, 2 \mathrm{~A}$ and $\Delta \mathrm{E}_{\mathrm{ads}}, 2 \mathrm{~B}$ the adsorption energy of the pure SAM consisting of 2 thiols $\mathrm{A}$ and $B$, on a $(\sqrt{ } 3 \times 2 \sqrt{ } 3) R 30$ o unit cell, respectively.

\begin{tabular}{|lll|}
\hline \multicolumn{2}{|l}{ Table 1.Brute formula and simplified nomenclature of the thyil radicals chosen to } \\
simulate SAMs of thiol molecules. \\
\hline Brute Formula & Simplified name & No. in Fig. 1 \\
\hline S- $(\mathrm{CH} 2) 11-\mathrm{CH} 3$ & $\mathrm{C} 12$ & 1 \\
S- $(\mathrm{CH} 2) 3-\mathrm{COOH}$ & $\mathrm{C} 3 \mathrm{COOH}$ & 2 \\
S- $(\mathrm{CH} 2) 11-\mathrm{COOH}$ & $\mathrm{C} 11 \mathrm{COOH}$ & 3 \\
$\mathrm{~S}-(\mathrm{CH} 2) 15-\mathrm{COOH}$ & $\mathrm{C} 15 \mathrm{COOH}$ & 4 \\
S- $(\mathrm{CH} 2) 5-\mathrm{CO}-\mathrm{NH}-\mathrm{CH} 3$ & $\mathrm{C} 5 \mathrm{CONHCH} 3$ \\
$\mathrm{~S}-(\mathrm{CH} 2) 2-\mathrm{O}-(\mathrm{CH} 2) 2-\mathrm{O}-(\mathrm{CH} 2) 2-\mathrm{O}-$ & $\mathrm{Eg}$ & 5 \\
$(\mathrm{CH} 2) 2-\mathrm{O}-\mathrm{CH} 3$ & & 6 \\
$\mathrm{~S}-(\mathrm{CH} 2) 5-\mathrm{CO}-\mathrm{NH}-(\mathrm{CH} 2) 2-\mathrm{O}-(\mathrm{CH} 2) 2-\mathrm{O}-$ & TriEg & 7 \\
$(\mathrm{CH} 2) 2-\mathrm{O}-\mathrm{CH} 3$ & &
\end{tabular}

\section{Experimental details}

All reagents, including MUA (11-mercaptoundecanoic acid), were purchased from SigmaAldrich and used as received.

\section{Nanoparticles synthesis}

The gold nanoparticles (AuNPs) were synthesized by the Turkevich's method. [65] $9.85 \mathrm{mg}$ of $\mathrm{HAuCl}_{4}, 3 \mathrm{H}_{2} \mathrm{O}$ (1.0 eq., $2.5 \cdot 10^{-5} \mathrm{~mol}$ ) were dissolved in $95 \mathrm{~mL}$ of water. The solution was boiled in a glass bottle, then $5 \mathrm{~mL}$ of a solution of trisodium citrate dihydrate 7.5.10- 
${ }^{3} \mathrm{~mol} \cdot \mathrm{L}^{-1}$ were added to the gold precursor solution under vigorous stirring. The mixture was heated for 30 minutes and cooled to room temperature.

\section{Ligand synthesis}

\section{TriEg :}

(N-\{2-[2-(2-methoxyethoxy)ethoxy]ethyl\}-6-sulfanylhexanamide) was synthesized using the method reported by Pengo et al.[66] with peptide coupling according to Kleinert et al. ${ }^{[67]}$ Eg was synthesized following a previously reported procedure.[68]

\section{Mixed SAMs formation on gold nanoparticles}

A suspension of $35 \mathrm{~nm}$ gold nanoparticles (initial gold concentration $[\mathrm{Au}]=2.5 \cdot 10^{-4} \mathrm{~mol} \cdot \mathrm{L}^{-}$

$\left.{ }^{1}\right)$ was gently centrifuged for 30 minutes at $3000 \mathrm{~g}$ and the supernatant was removed in order to reduce 6 times its initial volume. The concentrated suspension was divided into $500 \mu \mathrm{L}$ batches and $1500 \mu \mathrm{L}$ of absolute ethanol was added to each batch so that the final Au concentration in each aliquot was $[\mathrm{Au}]=3.75 \cdot 10^{-4} \mathrm{~mol} \cdot \mathrm{L}^{-1}$. Meanwhile, each ligand was solubilized separately in ethanol to yield $2.6 \cdot 10^{-4} \mathrm{~mol}^{-L^{-1}}$ solutions. Then, Eg or TriEg solutions were added to a gold nanoparticles aliquot, with a surface coverage of $3.5 \mathrm{ligand} \cdot \mathrm{nm}^{-2}$, and the thiocarboxylic acid $\mathrm{HS}(\mathrm{CH} 2) 3 \mathrm{COOH}$ or $\mathrm{HS}(\mathrm{CH} 2) 15 \mathrm{COOH}$ was added for a surface coverage of $1.5 \mathrm{acid} \cdot \mathrm{nm}^{-2}$. The reaction media in a closed vial were put in an oven at $40^{\circ} \mathrm{C}$ for 1 hour in order to allow reorganization of monolayers and eventual ligand exchange. The sample corresponding to the mixture of the longest chains Trieg and $\mathrm{HS}\left(\mathrm{CH}_{2}\right)_{15} \mathrm{COOH}$ was allowed to equilibrate for $5 \mathrm{~h}$.

\section{Silica heterogeneous nucleation and growth}

After equilibration, a $0.375 \mathrm{~mol} \cdot \mathrm{L}^{-1}$ ethanolic solution of tetraethylorthosilicate (TEOS) was added with a molar ratio Au:TEOS $=1: 2$ (except for the $\mathrm{Eg} / \mathrm{HS}(\mathrm{CH} 2) 15 \mathrm{COOH}$ for which Au:TEOS $=1: 1.5$ was used). $90 \mu \mathrm{L}$ of ammonia at $14.8 \mathrm{~mol} \cdot \mathrm{L}^{-1}$ was immediately added. The reaction medium was stirred manually between each addition and heated at $40^{\circ} \mathrm{C}$ for 15 hours. After centrifugation and redispersion in ethanol, a drop of the suspension was 
deposited and dried on a carbon-coated copper grid for further analyses by transmission electron microscopy (TEM) using a Tecnai Spirit 2 microscope operating at a $120 \mathrm{kV}$ voltage.

\section{Results and Discussion}

\section{Adsorption geometries and binding energies}

The adsorption geometries of the thiol SAMs are fully relaxed in a $(\sqrt{ } 3 \times 2 \sqrt{ } 3) \mathrm{R} 30$ o unit cell. Both thiols in the unit cell are found to adsorb on a displaced bridge site depending on the surface Au atom displacement due to the magnitude of the Au-S interaction. This result is in agreement with those observed for pure alkane thiols.[62] The tilt angle values for the different adsorbed thiolates are all within the range of $30-35^{\circ}$. The results can be compared with the recent work of Mete et al. on pure alkyl chain thiols. [69] Indeed, the tilt angle, interatomic distances, and adsorption energies are comparable taking into account the presence of extra dipole-dipole interactions, $\mathrm{H}$-bonds and other inter-chain interactions. A trend can clearly be deduced after qualitative extrapolation of the results.

From the $d(A u-S)$ distances (about $2.4 \AA$ in agreement with experiment[10]) and the vertical spacing between $S$ and the surface, one can calculate the largest distance $(\Delta z)$ between the $\mathrm{S}$ neighbouring $\mathrm{Au}$ atom and its original position in a clean $\mathrm{Au}(111)$ slab (Fig. 3 , Table 2). This descriptor is used to quantify and discuss the surface reconstruction.

\begin{tabular}{|c|c|c|c|}
\hline Thiolate & $\Delta \mathrm{E}_{\text {int.chain }}$ & $\Delta \mathrm{E}_{\text {recon }}$ & $\Delta \mathrm{z}$ \\
\hline $\mathrm{C} 5 \mathrm{CONHCH} 3$ & -0.83 & 0.11 & 0.15 \\
\hline TriEg & -1.49 & 0.16 & 0.23 \\
\hline $\mathrm{Eg}$ & -0.65 & 0.19 & 0.10 \\
\hline C3 & -0.01 & 0.08 & 0.03 \\
\hline C11 & -0.65 & 0.21 & 0.14 \\
\hline
\end{tabular}




\begin{tabular}{|llll|}
$\mathrm{C} 12$ & -0.72 & 0.31 & 0.13 \\
$\mathrm{C} 3 \mathrm{COOH}$ & -0.41 & 0.09 & 0.05 \\
$\mathrm{C} 11 \mathrm{COOH}$ & -0.90 & 0.24 & 0.23 \\
$\mathrm{C} 15 \mathrm{COOH}$ & -1.75 & 0.21 & 0.19 \\
\hline
\end{tabular}

Table 3. Adsorption and binding energy for the pure thiol SAMs calculated at the PBED3 level. (Energies in eV, Distances in Å)

\begin{tabular}{llll|}
\hline Thiolate & $\Delta \mathrm{E}_{\text {ads }}$ & $\Delta \mathrm{E}_{\text {ads, PBE }}$ & $\Delta \mathrm{E}_{\text {bind(s-Au) }}$ \\
$\mathrm{C} 5 \mathrm{CONHCH} 3$ & -3.49 & -1.90 & -2.65 \\
TriEg & -4.15 & -1.82 & -2.66 \\
$\mathrm{Eg}$ & -3.20 & -1.47 & -2.55 \\
$\mathrm{C} 3$ & -2.64 & -1.64 & -2.63 \\
$\mathrm{C} 11$ & -3.25 & -1.62 & -2.60 \\
$\mathrm{C} 12$ & -3.32 & -1.22 & -2.60 \\
$\mathrm{C} 3 \mathrm{COOH}$ & -3.04 & -0.94 & -2.63 \\
$\mathrm{C} 11 \mathrm{COOH}$ & -3.48 & -1.43 & -2.59 \\
$\mathrm{C} 15 \mathrm{COOH}$ & -4.39 & -1.86 & -2.64 \\
\hline
\end{tabular}

$\Delta z$ obtained after geometry optimization is associated to a barrier-less surface reconstruction mechanism. From Table 2 and Fig. 4 , it can be seen that $\Delta z$ correlates with $\Delta \mathrm{E}_{\text {int.chain }}$ in the low interaction energy range. The higher the inter-chain energy between the thiol chains, the larger the reconstruction of the surface. However, $\Delta z$ reaches a plateau at $0.20 \AA$, suggesting that further reconstruction is associated with an activation energy. At this elevation surface reconstruction is blocked and probably depends on the chemical nature and geometry of the thiol chain. Qualitatively (not highlighted in the figures), two types of small reconstruction occur: elevation of an Au atom of the bridge site and elevation of both atoms of the bridge site. These behaviours indicate that all thiols chains do not modify or reconstruct the $\mathrm{Au}(111)$ surface in the same way. 


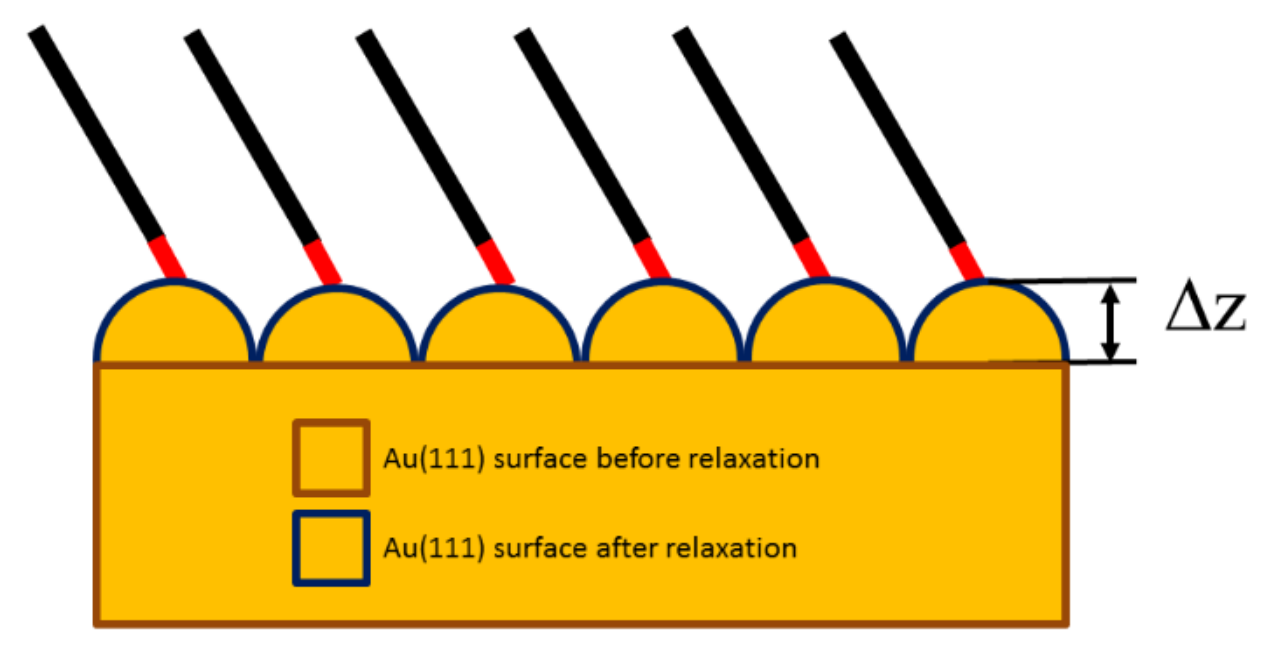

Figure 3. Schematic representation of the SAM structure showing the elevation distance of the upper layer atoms defined by $(\Delta z)$.

The reconstruction energy $\Delta \mathrm{E}_{\text {recon }}$ (See Eq. 6) was also calculated. Again, one could derive

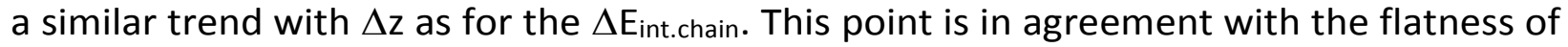
the potential energy surface for thiol adsorption on the $A u(111)$ surface. Hence, it is difficult to clearly see a correlation between $\mathrm{Au}$ atom displacement and interaction energy. It is clear that the next point to be investigated in detail at the DFT level is the study of the surface reconstruction potential energy surface and corresponding transition states.

The Au-S binding energy $\Delta \mathrm{E}_{\text {bind }}(\mathrm{S}-\mathrm{Au})$ was also calculated. Very interestingly $\Delta \mathrm{E}_{\text {bind }}(\mathrm{S}-\mathrm{Au})$ (See Eq. 4 ) is independent of the thiol chain in the series studied ( $\approx 2.6 \mathrm{eV}$ ), as illustrated by the blue bars in the graph of Figure 5. 


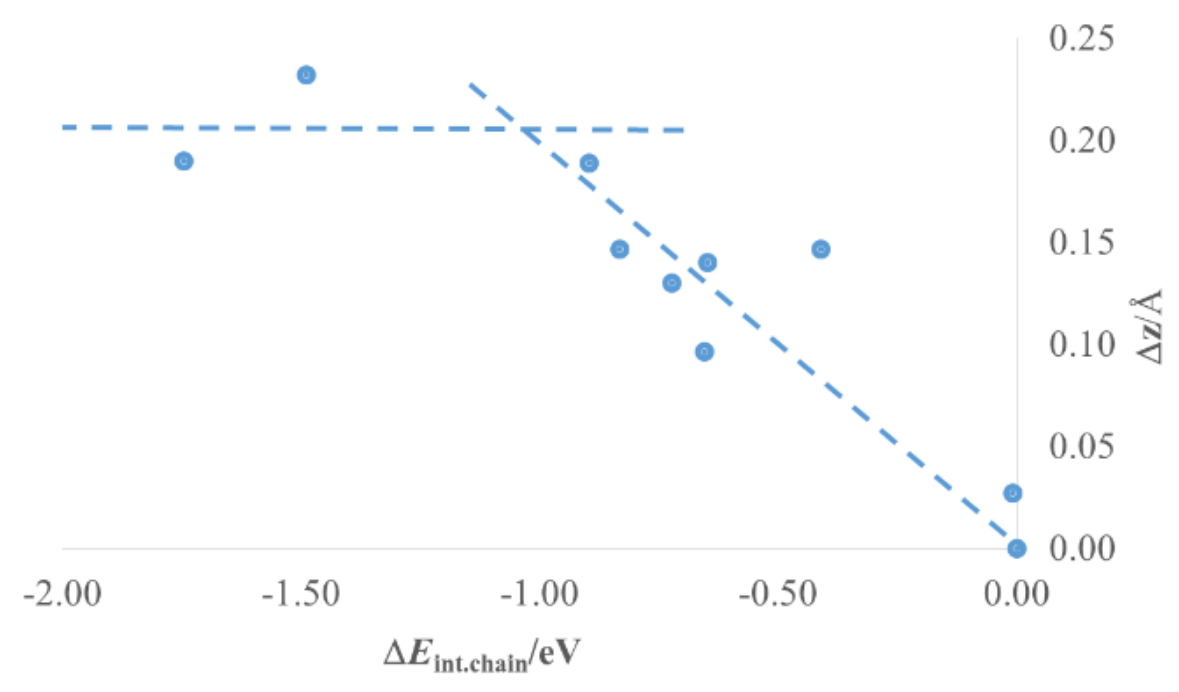

Figure 4. Relation between the PBE-D3 inter-chain interaction energy $\Delta \mathrm{E}_{\text {int-chain }}$ and the surface gold atom elevation $\Delta z$, showing a plateau around the maximum value $0.2 \AA$ for $\Delta z$. This border is considered to be associated with the activation energy needed to form a protrusion or adatom e.g. The dashed blue lines are drawn as guides to the eye.

Another descriptor that can describe thiol/Au(111) adsorption is the inter-chain interaction energy. In order to show its relevance one should be aware that in order to form an adatom, or another type of protrusion, on the surface, a critical $\Delta z$ should be reached (estimated above as $\sim 0.2 \AA$ ), followed by the crossing of the energy barrier for protrusion formation on the $\mathrm{Au}(111)$ surface. A linear behaviour between $\Delta \mathrm{z}$ and $\Delta_{\text {Eint-chain }}$ (Eq. 5) is highlighted in Figure 4, until the value of $\Delta z \approx 0.2 \AA$, after which a plateau is reached.

Surface reconstruction of the Au surfaces was previously described theoretically in long alkyl carboxylic acid and alcohol thiol SAMs[7, 35, 70, 71] and in carbenes[72, 73]. In order to reconstruct drastically the surface, strong binding to the Au surface is not enough. Earlier we have shown, using XPS and DFT, that short chain aminothiols[74] reconstruct more the surface than long chain thiols. So, the surface reconstruction reaction is dependent on the presence of geometrical constraints, i.e. steric hindrance. The geometry 
constraints in the longer chains will limit the reconstruction compared with the shorter ones, which are expected to pass the reconstruction energy barrier. This last statement has to be proven subsequently, however this is out of the present scope of this work, in which we focus mainly on the inter chain interactions in thiol/Au(111) SAMs.

\section{Adsorption energies}

The adsorption energies are calculated at the state-of-the-art PBE-D3 level (See Table 3) that takes into account dispersion interactions. Pure DFT-PBE energies $\left(\Delta \mathrm{E}_{\mathrm{ads}} \mathrm{PBE}\right)$ do not include dispersion interactions, then directly describe (a) the covalent S-Au binding energy and $(b)$ the pure electrostatic inter-chain interactions. Values of $\Delta E_{\text {bind }}(S-A u)$ confirm that for the pure SAM systems considered here, the Au-S contribution (See Table 3 and Fig. 5) is constant. The inclusion of dispersion correction is of outmost importance, as shown in Table 3. The values of the pure PBE adsorption energies are given for the sake of illustration. The role of dispersion becomes clear when the inter chain interaction energy is considered. It is the only component of the adsorption energy that is chain dependent, and thus dominates the adsorption trend. The effect of short alkyl thiol chain vs. long alkyl thiol chain, the effect of polar and nonpolar groups in the thiol chains, and the possibility to form $\mathrm{H}$-bonds between the thiol chains are clearly revealed and quantified only by using the PBE-D3 level of calculation. This quantification reproduces the alkyl thiol chain stability as a function of the alkyl chain length.[75]

Depending on the dominant type of inter-chain interaction (i.e. besides covalent interaction), the seven different thiols investigated can be divided in 3 families: $\mathrm{H}$-bond (C5CONHCH3 and TriEg), electrostatic (Eg), and mainly dispersion (C3, $\mathrm{C} 3 \mathrm{COOH}, \mathrm{C} 11, \mathrm{C} 12$, $\mathrm{C} 11 \mathrm{COOH}$, and $\mathrm{C} 15 \mathrm{COOH}$ ) interactions. While $\mathrm{C} 11 \mathrm{COOH}$, and $\mathrm{C} 15 \mathrm{COOH}$ can form $\mathrm{H}$-bonds with their neighbours, the dispersion interaction dominates the assembly. The $\mathrm{C} 3 \mathrm{COOH}$ thiol is the only chain that is weakly ordered, similar to the methylthiol SAM[76] due to the very low interaction forces between neighbouring chains. It is this property that probably enables a subsequently larger surface reconstruction thanks to the less 
constraint adsorption configurations at the surface. In Table 4 the effect of dispersion on the overall stability of the SAM can clearly be seen, e.g. the extremely low inter-chain stabilization for $\mathrm{C} 3 \mathrm{COOH}$ compared to the long alkyl chain $\mathrm{C} 15 \mathrm{COOH}$. Interesting to note is the high adsorption energy of the long chain thiols ( $\mathrm{C} 15 \mathrm{COOH}$ and TriEg), due to the strong inter chain interactions (See Table 2 and 3).

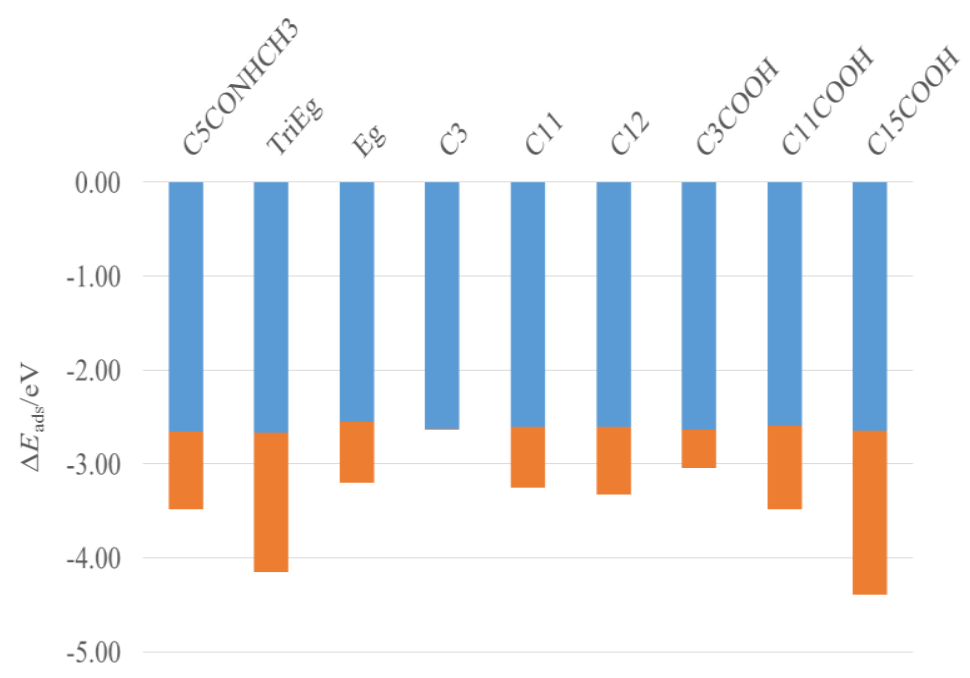

Figure 5. Decomposition of the adsorption energy, $\Delta E$ Eds, in $\mathrm{S}$-Au binding energy, $\Delta \mathrm{E}_{\text {bind, }}$ (blue) and inter chain interaction energy, $\Delta \mathrm{E}_{\text {int.chain, }}$ (orange) for thiol pair interactions. The total length of the bars indicates $\Delta \mathrm{E}_{\text {ads }}$ in $\mathrm{eV}$.

\section{Mixed SAMs: Prediction of the ligands segregation}

13 different and pertinent mixed SAM systems were investigated starting from our selection of thiols (See Table 1). The geometry of the mixed SAMs is not altered dramatically (i.e. adsorption site and tilting angle) compared with the pure ones.

The trend in adsorption energies deduced above for the pure SAMs is expected to be still valid, i.e. the dominance of inter-chain interactions. However, the interaction with different neighbouring thiol chains might deeply change the overall stability of the mixed SAMs. This cooperative effect is evaluated using a segregation descriptor $(\Xi)$, as defined 
in Eq. 7. From Table 5, one can divide the mixed SAMs in 3 groups: 1) those with $\Xi>0$ ) those with $\Xi \approx 0$, and those with $\Xi<0$, corresponding to 1 ) segregation, 2) no preference between pure and mixed, and 3) no segregation, respectively.

Table 4. Adsorption energy, dispersion energy, inter chain interaction energy for the different SAMs investigated at the PBE-D3 level. Energy values in eV.

\begin{tabular}{|lcccc|}
\hline SAMs & $\Delta$ Eads & $\Delta$ Eads. & $\Delta \mathrm{E}$ & $\Delta$ Eads. \\
\hline C12_C3COOH & & PBE & disp. & mean \\
C12_C11COOH & -3.03 & -1.61 & -1.42 & -3.18 \\
Eg_C3COOH & -3.38 & -1.37 & -2.01 & -3.40 \\
Eg_C15COOH & -2.97 & -1.63 & -1.34 & -3.12 \\
Eg_C11COOH & -3.72 & -1.66 & -2.06 & -3.80 \\
Eg_C12 & -3.27 & -1.39 & -1.88 & -3.34 \\
\hline TriEg_C3COOH & -3.30 & -1.35 & -1.95 & -3.26 \\
\hline TriEg_C11COOH & -2.95 & -1.75 & -1.20 & -3.60 \\
TriEg_C15COOH & -3.61 & -1.48 & -2.14 & -3.82 \\
TriEg_C12 & -4.04 & -1.74 & -2.30 & -4.27 \\
C15-COOH_C3COOH & -3.54 & -1.44 & -2.11 & -3.74 \\
C5CONHCH3_C12 & -3.44 & -1.91 & -1.53 & -3.71 \\
C5CONHCH3_ & -3.23 & -1.50 & -1.73 & -3.41 \\
C3COOH & -3.20 & -1.86 & -1.34 & -3.26 \\
\hline
\end{tabular}

Because the aim of this work is to identify ligands couples suitable to yield segregated SAMs on nanoparticles, we have focused on systems predicted to phase separate from Table 5, i.e. with positive $\Xi$ value. The only structure with a negative $\Xi$ value is calculated for the Eg_C12 mixture (-0.03 eV). However, this result should be taken with caution since the value is very close to 0 and probably close to the accuracy limits of the calculation level. The most segregating mixture is found for TriEg_C3COOH, which confirms the chemical intuition based on the structure of both ligands. This result is also very well 
reproduced by the experiment (see below). Of course, in order to check our $\Xi$ descriptor it would be interesting to look for nanoparticles having favourable mixing ligands on their surface.

Another interesting point to investigate is the shape of nanoparticles covered with short and less interacting thiol chains. Since short non-interacting ligands are expected to result in larger surface reconstruction than the longer and strongly interacting ligands, the shape of short ligand-covered NP should be different.

Nevertheless, it should be noted that the above mentioned descriptor $\Xi$, does not include entropy factors. Energetically, different distributions of the ligands at the surface differ mostly by their entropy. Especially, stabilization of the mixed SAM versus the pure SAM can occur by entropic effects. Configurational entropy (mixing entropy) [77-80] does not discriminate systems composed of mixtures of similar chains lengths, but might influence the results for systems composed of thiols chains with a large chain length differences.

Table 5. Comparison between the predicted segregation and the experimental observations for $1: 1$ thiol mixtures. ( $\Xi$ in $\mathrm{eV}$ )

\begin{tabular}{|llll|}
\hline SAM & $\Xi$ & Segregation & Exp. Obs. \\
\hline C12_C3COOH & 0.15 & YES & - \\
C12_C11COOH & 0.03 & YES/NO & - \\
Eg_C3COOH & 0.15 & YES & YES \\
Eg_C15COOH & 0.08 & YES & YES \\
Eg_C11COOH & 0.08 & YES & YES \\
Eg_C12 & -0.03 & NO/YES & - \\
TriEg_C3COOH & 0.65 & YES & YES \\
TriEg_C11COOH & 0.21 & YES & YES \\
TriEg_C15COOH & 0.23 & YES & YES \\
TriEg_C12 & 0.20 & YES & -
\end{tabular}




\begin{tabular}{|llll|} 
C15-COOH_C3COOH & 0.27 & YES & - \\
C5CONHCH3_C12 & 0.17 & YES & - \\
C5CONHCH3_C3COOH & 0.06 & YES/No & - \\
\hline
\end{tabular}

Segregation and comparison with experiment

Phase segregation in mixed SAMs was addressed on model hybrid nanoparticles consisting in a gold inorganic core functionalized with a mixed organic shell. Big particles of $35 \mathrm{~nm}$ are used with a sufficiently low surface curvature to allow direct comparison with the calculations on flat SAMs. Citrate-functionalized particles suspended in water were ligand-exchanged with a mixture of a polyethyleneglycol-terminated ligand (TriEg or $\mathrm{Eg})$ and a thiocarboxylic acid of varying chain length $(\mathrm{C} 3 \mathrm{COOH}$ or $\mathrm{C} 15 \mathrm{COOH})$ at a total ratio of 5 thiol.nm-2, enough to saturate the surface and avoid a large amount of free ligands in solution. $[1,81]$ To enhance electron contrast in the organic corona, silica was selectively nucleated and grown over carboxylic-rich domains, while areas rich in ethyleneglycol oligomers do not allow silica nucleation. Transmission electron microscopy (TEM,) images for the nanoparticles are shown in Figure 6.

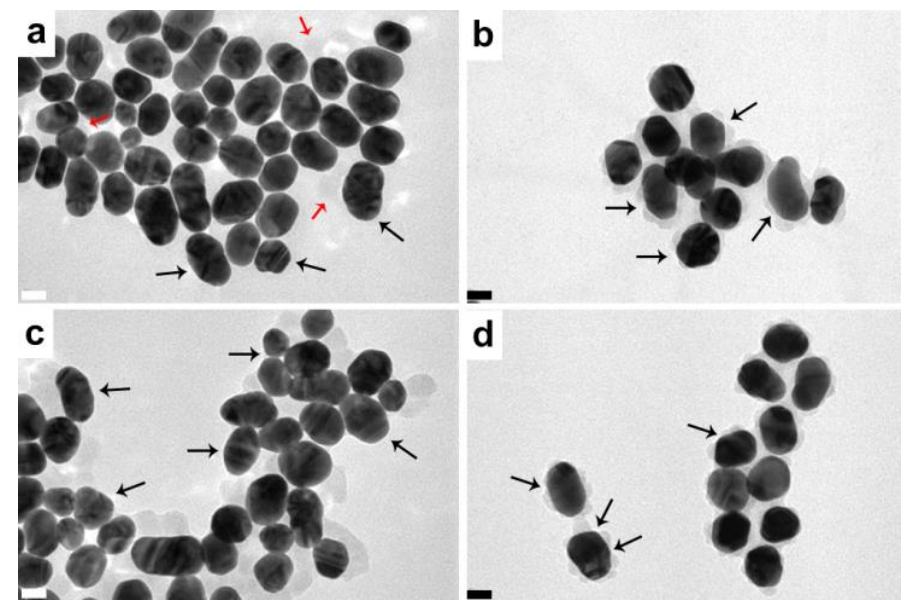

Figure 6. TEM pictures of $35 \mathrm{~nm}$ gold nanoparticles functionalized with mixed SAMs that are highlighted by silica growth on the carboxylic-rich domains. Non uniform shells and "holes" (arrows) highlight TriEg or Eg-rich domains, hence partial segregation in the mixed 
SAM:(a) TriEg/C3COOH, (b) TriEg/C15COOH, (c) Eg/C4COOH and (d) Eg/C15COOH. Molar ratio for all mixed SAMs: 3.5(TriEg-Eg):1.5(thiocarboxylic acid). Scale bars: $20 \mathrm{~nm}$.Red arrows in (a) highlight silica domains functionalized with mixed SAMs highlight silica patches and other areas free of silica. Hence, nanophase segregation occurs with the investigated mixed SAMs, in agreement with predictions from the calculated segregation parameter (Table 5).

\section{Discussion and conclusions}

Although the calculations have been performed on a defect free surface, which is an approximation of the more complex NP surface which contains different surfaces and especially steps and borders that are much more reactive, although proportionally less exposed, the quantum chemical calculations corrected for dispersion show a good agreement with experiment. It is true that experimentally the segregation is observed using silica adsorption, which expected not to alter the SAMs organization below. Having these approximations in mind we were able to describe the stability and segregation properties of binary mixtures of a series of thiol SAMs on $A u(111)$ investigated using periodic DFT and discussed compared with experimental observations.

The S-Au bond was calculated to be independent of the side chain type i.e. $\pm 2.6 \mathrm{eV}$ per thiol, showing that the thiol tail chain does not influence significantly the S-Au chemical properties for the studied linear chains. This finding is at least true for the alkane thiols since the S-Au bond energy has been found to be lower and also almost constant in the case of aryl thiols (i.e. $2.2 \mathrm{eV}$ ).[82, 83] This leads us to conclude that the S-Au bond is sensitive to the hybridization state if the carbon atom bound to the sulphur atom. However, this does not mean that the thiol tail does not matter. At the contrary, if one are interested in the mixing mechanisms of different thiols, it is clear that the interatomic interactions are dominating.

The energy decomposition scheme used in this study helped us to understand the competitions that are at play in thiol SAM formation and confirms our intuition that the 
thiol SAMs do not all adsorb in the same configuration and do not all reconstruct the surface in the same way[74]. This point was also very concluded in our recent investigation on the thiol SAM formation reaction paths analysis.[36]

The surface is indeed found to be affected by adsorption of the thiol radicals. No barrierless adatom formation was observed, however Au-atoms were pulled out of the surface. This phenomenon was already describe by us some time ago[70] and reconfirmed experimentally later[74]. Interesting to note is that a relation between the inter-chain interaction energy and the elevation $(\Delta z)$ of the Au surface is found, i.e. in order to have protrusions formed that are elevated with a $\Delta z$ larger than $0.20 \AA$, an energy barrier should be crossed. This is deduced from the fact that $0.20 \AA$ is the maximum value and that we expect adatom formation in some cases. However, here to investigate this phenomenon in detail, a reaction path analysis should be performed, i.e. the calculation of the associated energy barrier for the adatom formation.

A segregation parameter was defined to characterize mixed thiol SAMs. With this parameter we are now able to predict, within the approximation that entropy driven segregation is negligible, the formation or not of segregated patches on a Au surface, as confirmed with experiment on nanoparticles. The most segregating mixture of our selection is found for TriEg_C3COOH. The least segregating mixtures are found or predicted for C12_C11COOH and C12_Eg.

In this study we have discussed and rationalized the interaction affinity between different linear thiols based on an adsorption energy decomposition strategy. This procedure is promising to identify quickly future segregated SAMs to drive the synthesis of e.g. nanoparticles with different surface phase segregation, ultimately leading to patchy or even Janus nanoparticles.

\section{Acknowledgements}

D.N. acknowledges the Islamic Development BankID number 600027536 for a postdoctoral grant. This work was performed using $\mathrm{HPC}$ resources from $\mathrm{GENCl}$ [CCRT/CINES/IDRIS] (Grant 2015-[x2015082022]) and the CCRE of Université Pierre et 
Marie Curie. C.G., C.C., C.S. and D.P. thank the C'Nano Ile de France DIM Nano-K for funding under the project $\mathrm{NaJaH}$, and Thomas Marchandier for experimental support.

\section{References}

[1] J.C. Love, L.A. Estroff, J.K. Kriebel, R.G. Nuzzo, G.M. Whitesides, Self-assembled monolayers of thiolates on metals as a form of nanotechnology, Chemical Reviews, 105 (2005) 1103-1169.

[2] T.D. Dunbar, M.T. Cygan, L.A. Bumm, G.S. McCarty, T.P. Burgin, W.A. Reinerth, L. Jones, J.J. Jackiw, J.M. Tour, P.S. Weiss, D.L. Allara, Combined scanning tunneling microscopy and infrared spectroscopic characterization of mixed surface assemblies of linear conjugated guest molecules in host alkanethiolate monolayers on gold, Journal of Physical Chemistry B, 104 (2000) 4880-4893.

[3] R.K. Smith, P.A. Lewis, P.S. Weiss, Patterning self-assembled monolayers, Progress in Surface Science, 75 (2004) 1-68.

[4] C.D. Bain, E.B. Troughton, Y.T. Tao, J. Evall, G.M. Whitesides, R.G. Nuzzo, Formation of Monolayer Films by the Spontaneous Assembly of Organic Thiols from Solution onto Gold, Journal of the American Chemical Society, 111 (1989) 321-335.

[5] J.P. Folkers, P.E. Laibinis, G.M. Whitesides, Self-Assembled Monolayers of Alkanethiols on Gold Comparisons of Monolayers Containing Mixtures of Short-Chain and Long-Chain Constituents with Ch3 and Ch2oh Terminal Groups, Langmuir, 8 (1992) 1330-1341.

[6] J.P. Folkers, P.E. Laibinis, G.M. Whitesides, J. Deutch, Phase-Behavior of 2-Component Self-Assembled Monolayers of Alkanethiolates on Gold, Journal of Physical Chemistry, 98 (1994) 563-571.

[7] F. Tielens, V. Humblot, C.-M. Pradier, Exploring the reactivity of mixed omega-functionalized undecanethiol self-assembled monolayers - A DFT study, International Journal of Quantum Chemistry, 108 (2008) 1792-1795.

[8] A. Santos, J.A. Millan, S.C. Glotzer, Facetted patchy particles through entropy-driven patterning of mixed ligand SAMS, Nanoscale, 4 (2012) 2640-2650.

[9] T. Sawaguchi, Y. Sato, F. Mizutani, In situ STM imaging of individual molecules in two-component selfassembled monolayers of 3-mercaptopropionic acid and 1-decanethiol on $\mathrm{Au}(111)$, Journal of Electroanalytical Chemistry, 496 (2001) 50-60.

[10] I. Hehn, S. Schuster, T. Waechter, T. Abu-Husein, A. Terfort, M. Zharnikov, E. Zojer, Employing X-ray Photoelectron Spectroscopy for Determining Layer Homogeneity in Mixed Polar Self-Assembled Monolayers, Journal of Physical Chemistry Letters, 7 (2016) 2994-3000.

[11] P.G. De Gennes, Noble Lecture, in, 1991.

[12] A. Walther, A.H.E. Mueller, Janus particles, Soft Matter, 4 (2008) 663-668.

[13] D. Suzuki, H. Kawaguchi, Janus particles with a functional gold surface for control of surface plasmon resonance, Colloid and Polymer Science, 284 (2006) 1471-1476.

[14] S.-H. Kim, S.-J. Jeon, W.C. Jeong, H.S. Park, S.-M. Yang, Optofluidic Synthesis of Electroresponsive Photonic Janus Balls with Isotropic Structural Colors, Advanced Materials, 20 (2008) 4129-4134.

[15] M.D. McConnell, M.J. Kraeutler, S. Yang, R.J. Composto, Patchy and Multiregion Janus Particles with Tunable Optical Properties, Nano Letters, 10 (2010) 603-609.

[16] J.N. Anker, R. Kopelman, Magnetically modulated optical nanoprobes, Applied Physics Letters, 82 (2003) 1102-1104.

[17] C.J. Behrend, J.N. Anker, B.H. McNaughton, M. Brasuel, M.A. Philbert, R. Kopelman, Metal-capped Brownian and magnetically modulated optical nanoprobes (MOONs): Micromechanics in chemical and biological microenvironments, Journal of Physical Chemistry B, 108 (2004) 10408-10414. 
[18] J.-W. Kim, D. Lee, H.C. Shum, D.A. Weitz, Colloid surfactants for emulsion stabilization, Advanced Materials, 20 (2008) 3239-+.

[19] N. Glaser, D.J. Adams, A. Boeker, G. Krausch, Janus particles at liquid-liquid interfaces, Langmuir, 22 (2006) 5227-5229.

[20] A. Synytska, R. Khanum, L. Ionov, C. Cherif, C. Bellmann, Water-Repellent Textile via Decorating Fibers with Amphiphilic Janus Particles, Acs Applied Materials \& Interfaces, 3 (2011) 1216-1220.

[21] J.R. Millman, K.H. Bhatt, B.G. Prevo, O.D. Velev, Anisotropic particle synthesis in dielectrophoretically controlled microdroplet reactors, Nature Materials, 4 (2005) 98-102.

[22] C. Vilain, F. Goettmann, A. Moores, P. Le Floch, C. Sanchez, Study of metal nanoparticles stabilised by mixed ligand shell: a striking blue shift of the surface-plasmon band evidencing the formation of Janus nanoparticles, Journal of Materials Chemistry, 17 (2007) 3509-3514.

[23] F. Cardinaux, A. Stradner, P. Schurtenberger, F. Sciortino, E. Zaccarelli, Modeling equilibrium clusters in lysozyme solutions, Epl, 77 (2007).

[24] E. Sanz, C. Valeriani, T. Vissers, A. Fortini, M.E. Leunissen, A. van Blaaderen, D. Frenkel, M. Dijkstra, Out-of-equilibrium processes in suspensions of oppositely charged colloids: liquid-to-crystal nucleation and gel formation, Journal of Physics-Condensed Matter, 20 (2008).

[25] E. Zaccarelli, S.M. Liddle, W.C.K. Poon, On polydispersity and the hard sphere glass transition, Soft Matter, 11 (2015) 324-330.

[26] J. Colombo, M. Dijkstra, Effect of quenched size polydispersity on the fluid-solid transition in charged colloidal suspensions, Journal of Chemical Physics, 134 (2011).

[27] A.M. Somoza, E. Chacon, L. Mederos, P. Tarazona, A MODEL FOR MEMBRANES, VESICLES AND MICELLES IN AMPHIPHILIC SYSTEMS, Journal of Physics-Condensed Matter, 7 (1995) 5753-5776.

[28] Z.Y. Liu, R.H. Guo, G.X. Xu, Z.H. Huang, L.T. Yan, Entropy-Mediated Mechanical Response of the Interfacial Nanoparticle Patterning, Nano Letters, 14 (2014) 6910-6916.

[29] C. Ma, H. Wu, Z.H. Huang, R.H. Guo, M.B. Hu, C. Kubel, L.T. Yan, W. Wang, A Filled-HoneycombStructured Crystal Formed by Self-Assembly of a Janus Polyoxometalate-Silsesquioxane (POM-POSS) CoCluster, Angewandte Chemie-International Edition, 54 (2015) 15699-15704.

[30] D. Costa, M. Smerieri, I. Tranca, L. Savio, L. Vattuone, F. Tielens, DFT Atomistic Thermodynamics Applied To Elucidate the Driving Force behind Glutamic Acid Self-Assemblies on Silver (100) Surface, Journal of Physical Chemistry C, 118 (2014) 29874-29879.

[31] M. Smerieri, L. Vattuone, D. Costa, F. Tielens, L. Savio, Self-Assembly of (S)-Glutamic Acid on Ag(100): A Combined LT-STM and Ab Initio Investigation, Langmuir, 26 (2010) 7208-7215.

[32] I. Tranca, M. Smerieri, L. Savio, L. Vattuone, D. Costa, F. Tielens, Unraveling the Self-Assembly of the (S)-Glutamic Acid "Flower" Structure on Ag(100), Langmuir, 29 (2013) 7876-7884.

[33] V. Humblot, F. Tielens, N.B. Luque, H. Hampartsoumian, C. Methivier, C.-M. Pradier, Characterization of Two-Dimensional Chiral Self-Assemblies L- and D-Methionine on Au(111), Langmuir, 30 (2014) 203-212.

[34] F. Tielens, V. Humblot, C.-M. Pradier, Elucidation of the low coverage chiral adsorption assembly of L-lysine on Cu(110) surface: A theoretical study, Surface Science, 602 (2008) 1032-1039.

[35] F. Tielens, E. Santos, AuS and SH Bond Formation/Breaking during the Formation of Alkanethiol SAMs on $\mathrm{Au}(111)$ : A Theoretical Study, Journal of Physical Chemistry C, 114 (2010) 9444-9452.

[36] H. Guesmi, N.B. Luque, E. Santos, F. Tielens, Does the S-H Bond Always Break after Adsorption of a Alkylthiol on Au(111)?, Chem. Eur. J., 23 (2016) 1402-1408.

[37] A.D. Souza, I. Rungger, R.B. Pontes, A.R. Rocha, A.J.R. da Silva, U. Schwingenschlogl, S. Sanvito, Stretching of BDT-gold molecular junctions: thiol or thiolate termination?, Nanoscale, 6 (2014) 1449514507.

[38] F. Schreiber, Structure and growth of self-assembling monolayers, Progress in Surface Science, 65 (2000) 151-256. 
[39] H. Hakkinen, M. Walter, H. Gronbeck, Divide and protect: Capping gold nanoclusters with molecular gold-thiolate rings, Journal of Physical Chemistry B, 110 (2006) 9927-9931.

[40] P. Maksymovych, D.C. Sorescu, J.T. Yates, Gold-adatom-mediated bonding in self-assembled shortchain alkanethiolate species on the Au(111) surface, Physical Review Letters, 97 (2006).

[41] A. Bencini, G. Rajaraman, F. Totti, M. Tusa, Modeling thiols on Au(111): Structural, thermodynamic and magnetic properties of simple thiols and thiol-radicals, Superlattices and Microstructures, 46 (2009) 4-9.

[42] G. Rajaraman, A. Caneschi, D. Gatteschi, F. Totti, A periodic mixed gaussians-plane waves DFT study on simple thiols on $\mathrm{Au}(111)$ : adsorbate species, surface reconstruction, and thiols functionalization, Physical Chemistry Chemical Physics, 13 (2011) 3886-3895.

[43] G. Kresse, J. Furthmuller, Efficient iterative schemes for ab initio total-energy calculations using a plane-wave basis set, Physical Review B, 54 (1996) 11169-11186.

[44] G. Kresse, D. Joubert, From ultrasoft pseudopotentials to the projector augmented-wave method, Physical Review B, 59 (1999) 1758-1775.

[45] J.P. Perdew, K. Burke, M. Ernzerhof, Generalized gradient approximation made simple, Physical Review Letters, 77 (1996) 3865-3868.

[46] J.P. Perdew, K. Burke, M. Ernzerhof, Generalized gradient approximation made simple (vol 77, pg 3865, 1996), Physical Review Letters, 78 (1997) 1396-1396.

[47] F. Tielens, C. Gervais, J.F. Lambert, F. Mauri, D. Costa, Ab initio study of the hydroxylated surface of amorphous silica: A representative model, Chemistry of Materials, 20 (2008) 3336-3344.

[48] M. Calatayud, F. Tielens, F. De Proft, Reactivity of gas-phase, crystal and supported V2O5 systems studied using density functional theory based reactivity indices, Chemical Physics Letters, 456 (2008) 5963.

[49] T.V. de Bocarme, T.D. Chau, F. Tielens, J. Andres, P. Gaspard, R.L.C. Wang, H.J. Kreuzer, N. Kruse, Oxygen adsorption on gold nanofacets and model clusters, Journal of Chemical Physics, 125 (2006).

[50] F. Tielens, J. Andres, T.D. Chau, T.V. de Bocarme, N. Kruse, P. Geerlings, Molecular oxygen adsorption on electropositive nano gold tips, Chemical Physics Letters, 421 (2006) 433-438.

[51] F. Tielens, M. Calatayud, S. Dzwigaj, M. Che, What do vanadium framework sites look like in redox model silicate zeolites?, Microporous and Mesoporous Materials, 119 (2009) 137-143.

[52] F. Tielens, M. Trejda, M. Ziolek, S. Dzwigaj, Nature of vanadium species in V substituted zeolites: A combined experimental and theoretical study, Catalysis Today, 139 (2008) 221-226.

[53] P.E. Blochl, O. Jepsen, O.K. Andersen, Improved tetrahedron method for Brillouin-zone integrations, Physical Review B, 49 (1994) 16223-16233.

[54] P.E. Blochl, Projector Augmented-Wave Method, Physical Review B, 50 (1994) 17953-17979.

[55] S. Grimme, Semiempirical GGA-type density functional constructed with a long-range dispersion correction, Journal of Computational Chemistry, 27 (2006) 1787-1799.

[56] S. Grimme, J. Antony, S. Ehrlich, H. Krieg, A consistent and accurate ab initio parametrization of density functional dispersion correction (DFT-D) for the 94 elements H-Pu, Journal of Chemical Physics, 132 (2010).

[57] K. Toyoda, I. Hamada, K. Lee, S. Yanagisawa, Y. Morikawa, Density functional theoretical study of pentacene/noble metal interfaces with van der Waals corrections: Vacuum level shifts and electronic structures, Journal of Chemical Physics, 132 (2010).

[58] J. Slawinska, P. Dabrowski, I. Zasada, Doping of graphene by a Au(111) substrate: Calculation strategy within the local density approximation and a semiempirical van der Waals approach, Physical Review B, 83 (2011).

[59] C. Vericat, M.E. Vela, G.A. Benitez, J.A. Matin Gago, X. Torrelles, R.C. Salvarezza, J. Phys.: Condens. Matter, 18 (2006) R867.

[60] F. Schreiber, J. Phys.: Condens. Matter, 16 (2004) R881. 
[61] C. Vericat, M.E. Vela, R.C. Salvarezza, Self-assembled monolayers of alkanethiols on Au(111): surface structures, defects and dynamics, Physical Chemistry Chemical Physics, 7 (2005) 3258-3268.

[62] N.B. Luque, E. Santos, J. Andres, F. Tielens, Effect of Coverage and Defects on the Adsorption of Propanethiol on Au(111) Surface: A Theoretical Study, Langmuir, 27 (2011) 14514-14521.

[63] B. Diawara, ModelView, in.

[64] X. Wu, M.C. Vargas, S. Nayak, V. Lotrich, G. Scoles, Towards extending the applicability of density functional theory to weakly bound systems, Journal of Chemical Physics, 115 (2001) 8748-8757.

[65] J. Turkevich, P.C. Stevenson, J. Hillier, A STUDY OF THE NUCLEATION AND GROWTH PROCESSES IN THE SYNTHESIS OF COLLOIDAL GOLD, Discussions of the Faraday Society, (1951) 55-\&.

[66] P. Pengo, S. Polizzi, M. Battagliarin, L. Pasquato, P. Scrimin, Synthesis, characterization and properties of water-soluble gold nanoparticles with tunable core size, Journal of Materials Chemistry, 13 (2003) 24712478.

[67] M. Kleinert, T. Winkler, A. Terfort, T.K. Lindhorst, A modular approach for the construction and modification of glyco-SAMs utilizing 1,3-dipolar cycloaddition, Organic \& Biomolecular Chemistry, 6 (2008) 2118-2132.

[68] E.E. Foos, A.W. Snow, M.E. Twigg, M.G. Ancona, Thiol-terminated Di-, Tri-, and tetraethylene oxide functionalized gold nanoparticles: A water-soluble, charge-neutral cluster, Chemistry of Materials, 14 (2002) 2401-2408.

[69] E. Mete, M. Yortanlı, M.F. Danisman, A van der Waals DFT study of chain length dependence of alkanethiol adsorption on $\mathrm{Au}(111)$ : Physisorption vs. chemisorption, Phys. Chem. Chem. Phys, in press (2017).

[70] F. Tielens, D. Costa, V. Humblot, C.M. Pradier, Characterization of omega-functionalized undecanethiol mixed self-assembled monolayers on $\mathrm{Au}(111)$ : A combined polarization modulation infrared reflection-absorption spectroscopy/X-ray photoelectron spectroscopy/periodic density functional theory study, Journal of Physical Chemistry C, 112 (2008) 182-190.

[71] F. Tielens, V. Humblot, C.M. Pradier, M. Calatayud, F. Illas, Stability of Binary SAMs Formed by omegaAcid and Alcohol Functionalized Thiol Mixtures, Langmuir, 25 (2009) 9980-9985.

[72] M. Rodriguez-Castillo, D. Laurencin, F. Tielens, A. van der Lee, S. Clement, Y. Guari, S. Richeter, Reactivity of gold nanoparticles towards N-heterocyclic carbenes, Dalton Transactions, 43 (2014) 59785982.

[73] M. Rodríguez-Castillo , G. Lugo-Preciado, D. Laurencin, F. Tielens, A. van der Lee, S. Clément, Y. Guari, J.M. López-de-Luzuriaga, M. Monge, F. Remacle, S. Richeter, Experimental and Theoretical Study of the Reactivity of Gold Nanoparticles Towards Benzimidazole-2-ylidene Ligands, Chem. Eur. J., 22 (2016) 10446-10458.

[74] E. Bedford, V. Humblot, C. Methivier, C.-M. Pradier, F. Gu, F. Tielens, S. Boujday, An Experimental and Theoretical Approach to Investigate the Effect of Chain Length on Aminothiol Adsorption and Assembly on Gold, Chemistry-a European Journal, 21 (2015) 14555-14561.

[75] C. Vericat, M.E. Vela, G. Benitez, P. Carro, R.C. Salvarezza, Self-assembled monolayers of thiols and dithiols on gold: new challenges for a well-known system, Chemical Society Reviews, 39 (2010) 1805-1834.

[76] V. De Renzi, R. Di Felice, D. Marchetto, R. Biagi, U. del Pennino, A. Selloni, Ordered ( $3 \times 4)$ high-density phase of methylthiolate on Au(111), Journal of Physical Chemistry B, 108 (2004) 16-20.

[77] P.K. Ghorai, S.C. Glotzer, Atomistic Simulation Study of Striped Phase Separation in Mixed-Ligand SelfAssembled Monolayer Coated Nanoparticles, Journal of Physical Chemistry C, 114 (2010) 19182-19187.

[78] X. Liu, M. Yu, H. Kim, M. Mameli, F. Stellacci, Determination of monolayer-protected gold nanoparticle ligand-shell morphology using NMR, Nature Communications, 3 (2012).

[79] H. Kim, R.P. Carney, J. Reguera, Q.K. Ong, X. Liu, F. Stellacci, Synthesis and Characterization of Janus Gold Nanoparticles, Advanced Materials, 24 (2012) 3857-3863. 
[80] X.W. Ge, P.C. Ke, T.P. Davis, F. Ding, A Thermodynamics Model for the Emergence of a Stripe-like Binary SAM on a Nanoparticle Surface, Small, 11 (2015) 4894-4899.

[81] H. Hinterwirth, S. Kappel, T. Waitz, T. Prohaska, W. Lindner, M. Laemmerhofer, Quantifying Thiol Ligand Density of Self-Assembled Monolayers on Gold Nanoparticles by Inductively Coupled Plasma-Mass Spectrometry, Acs Nano, 7 (2013) 1129-1136.

[82] L.F. Peiretti, P. Quaino, F. Tielens, The Competition between Two High Density Assemblies of Poly Phenyl Thiols on Au(111), J. Phys. Chem. C, 120 (2016) 25462-25472.

[83] C. Goldmann, F. Ribot, L.F. Peiretti, P. Quaino, F. Tielens, C. Sanchez, C. Chanéac, D. Portehault, Quantified Binding Scale of Competing Ligands at the Surface of Gold Nanoparticles: The Role of Entropy and Intermolecular Forces, Small, (2017) 10.1002/smll.201604028. 
Douga Nassoko, Mahamadou Seydou, Claire Goldmann, Corinne Chanéac, Clément Sanchez, David Portehault, and Frederik Tielens

Rationalizing the formation of Binary Mixed Thiol Self-Assembled Monolayers

TEXT

Mixed thiolate Self Assembled Monolayers (SAMs) are used as a way toward adjusted surface functionalities for many applications. Nevertheless, geometrical and energetical properties, such as the ability to generate segregated domains and surface adatoms, is rather limited. This study provides for the first time an atomistic detailed picture of the interaction with the substrate and between the chains of different types of linear thiols from first principles.

GRAPHICAL ABSTRACT FIGURE

Prediction of the Mixing Behavior of Thiol Linkers on Au(111)

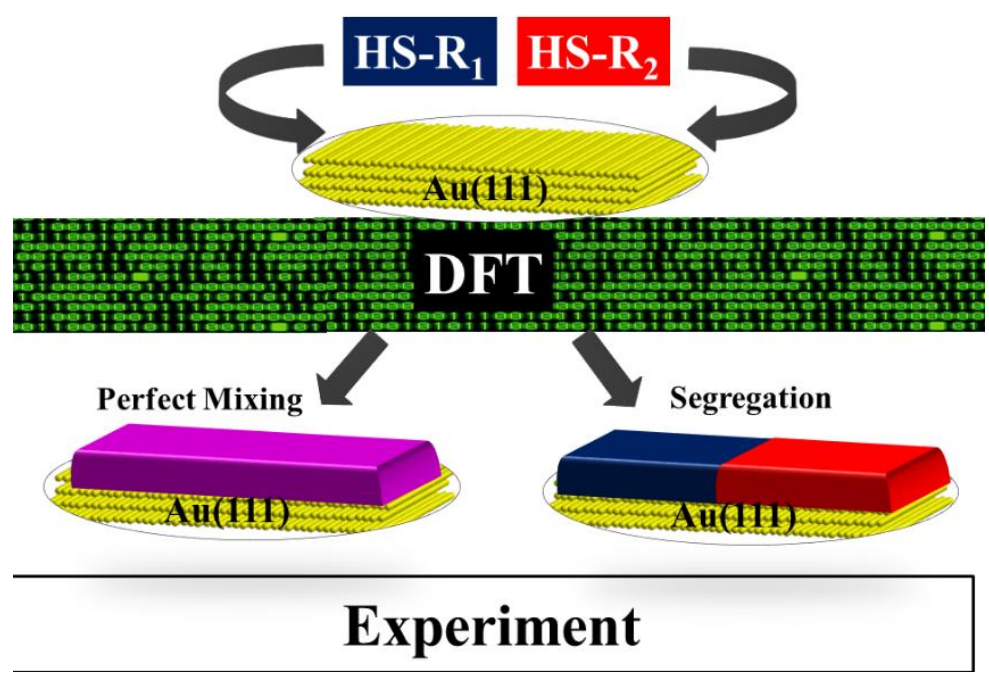

